\title{
Parallel Methods for the Numerical Integration of Ordinary Differential Equations
}

\author{
By Willard L. Miranker and Werner Liniger
}

Abstract. In this paper we derive a class of numerical integration formulas of a parallel type for ordinary differential equations. These formulas may be used simultaneously on a set of arithmetic processors to increase the integration speed. Conditions for the convergence of such formulas are formulated. Explicit examples for two and four processor cases are derived. Results of numerical experiments are given which show an effective improvement in computation speed.

0. Introduction. In this paper we consider the problem of speeding up the numerical integration of an ordinary differential equation. We suppose that we have a computer with a number of arithmetic processors capable of simultaneous operation and seek to devise parallel integration algorithms for execution on such a computer.

We do not address the combinatorial question of programming a calculation into independent segments which can be parcelled out among the processors. Instead we obtain new integration formulas which can be executed in parallel on a set of arithmetic processors and thereby increase the integration speed.

In $\$ 1$ we introduce the notion of computation front and describe the basic idea for broadening the front to allow for parallel computation. In $\$ 2$ we give a general formulation of a class of parallel integration methods of the linear multistep type for any even number of arithmetic processors. We also formulate sufficient conditions for the convergence of such numerical methods. In $\$ 3$ we apply the theory introduced in $\$ 2$ and derive explicit classes as well as individual examples of integration methods. In $\$ 4$ we present a discussion of the merits of various integration methods and give the results of numerical experiments with certain of the parallel methods derived in $\$ 3$. These results show effective gains in computation speeds. In an appendix we consider Runge-Kutta formulas which can be used in a parallel mode.

1. Description of the Numerical Method. The numerical integration of the initial-value problem for an ordinary differential equation $y^{\prime}=f(x, y)$ by finite differences is a sequential calculation. By this we mean that the approximation to the solution of an ordinary differential equation obtained by methods like linear multistep or Runge-Kutta evolves one point at a time. The solution at each new mesh point is a prescribed function of the values of the solution at certain previous mesh points.

Suppose that it is essential to find a numerical solution with a prescribed accuracy and to do so as quickly as possible. Suppose that a number of arithmetic processors are available with which to perform the rapid solution. Apart from the fact that in some cases we can parcel out the evaluation of $f(x, y)$ at a mesh point to

Received July 15, 1966. Revised November 15, 1966. 
make some use of the many processors at our disposal, it appears at first sight that the sequential nature of the numerical methods do not permit a parallel computation on all of the processors to be performed.

We say for the method of computation of the type being discussed, that the front of computation is too narrow to take advantage of more than one processor. (We neglect the possibility of parcelling out the evaluation of $f$ as beside our main objective.)

As a first step toward developing a parallel algorithm for the numerical solution of the initial-value problem, let us consider how we might widen the computation front. The predictor-corrector method of numerical integration provides a means for doing this. In its usual form this method of numerical integration is just as sequential as the single integration formula, and is not at first sight amenable to parallel computation on a number of arithmetic processors. Nevertheless, a slight alteration in the predictor-corrector mode will allow a parallel calculation.

We will now illustrate this with a simple example. For the problem

$$
y^{\prime}=f(x, y), \quad x>0, \quad y(0)=y_{0},
$$

we lay down a mesh of increment $h$. The mesh points are $x_{n}=(n-1) h, n=1,2$, $\cdots ; y_{n}$ denotes an approximation to $y$ at $x_{n} . y_{n}{ }^{p}$ and $y_{n}{ }^{c}$ are respectively the predicted and corrected values of $y_{n} . f_{n}{ }^{c}$ denotes $f\left(x_{n}, y_{n}{ }^{c}\right)$ and $f_{n}{ }^{p}$ denotes $f\left(x_{n}, y_{n}{ }^{p}\right)$. Consider the predictor-corrector formulas

$$
y_{n+1}^{p}=y_{n}{ }^{c}+\frac{h}{2}\left[3 f_{n}^{c}-f_{n-1}^{c}\right], \quad y_{n+1}^{c}=y_{n}{ }^{c}+\frac{h}{2}\left[f_{n+1}^{p}+f_{n}^{c}\right] .
$$

The sequence of computation is $\rightarrow y_{n+1}^{p} \rightarrow f_{n+1}^{p} \rightarrow y_{n+1}^{c} \rightarrow f_{n+1}^{c} \rightarrow$. This is schematized in the following diagram

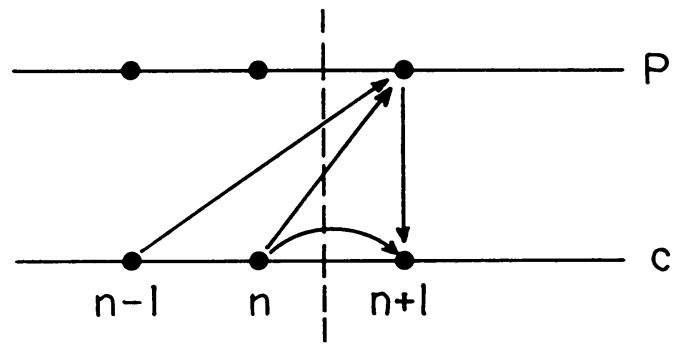

The upper line represents the progress of the computation at the mesh points for $y_{n}{ }^{p}$ and $f_{n}{ }^{p}$, the lower for $y_{n}{ }^{c}$ and $f_{n}{ }^{c}$. The broken vertical line is the computation front. The arrows indicate influences in the computation at the $(n+1)$ st mesh point and show that calculations ahead of the front depend on information on both sides of the front. This is characteristic of a sequential calculation.

A parallel mode of numerical integration is given by the following pair of formulas

$$
y_{n+1}^{p}=y_{n-1}^{c}+2 h f_{n}{ }^{p}, \quad y_{n}{ }^{c}=y_{n-1}^{c}+\frac{h}{2}\left(f_{n}^{p}+f_{n-1}^{c}\right) .
$$

Here the sequence of computation is schematized in the following diagram 


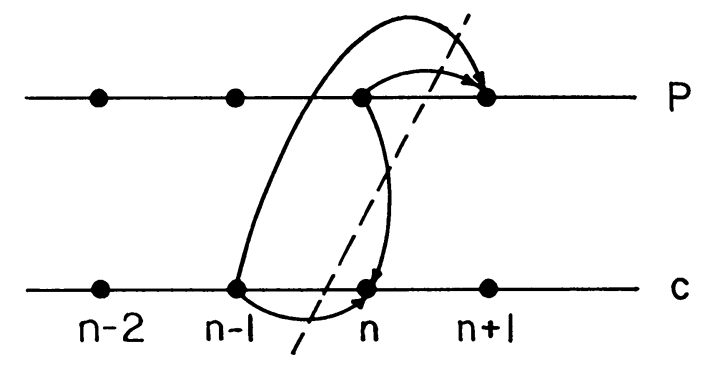

The arrows in the diagram show that computations at points ahead of the front depend only on information behind the front. This is characteristic of a parallel calculation. The sequence of computation is divided and each of its two parts

$$
y_{n+1}^{p} \rightarrow f_{n+1}^{p} \rightarrow, \quad y_{n}{ }^{c} \rightarrow f_{n}{ }^{c} \rightarrow
$$

may be simultaneously executed on separate arithmetic processors.

2. General Formulation. In this section we describe a general class of numerical integration methods which can be executed in parallel on $2 s$ processors, $s=1,2, \cdots$. We will also state and prove a theorem which guarantees the convergence of the solution of such a $2 s$ computer method to the solution of the differential equation as the mesh increment tends to zero. Minor modifications of our proof furnish convergence theorems for standard predictor-corrector methods as well as numerical methods for vector systems in which different equations in the system are approximated by different difference equations or by different combinations of predictorcorrector pairs or single open formulas.

Corresponding to the initial value problem (1.1), a $2 s$ computer method, $s=1$, $2, \cdots$, of parallel integration may be defined as follows. Let $\mathbf{y}_{\nu}$ be an $s$-vector,

$$
\mathbf{y}_{\nu}=\left(y_{\nu s}, \cdots, y_{(\nu-1) s+1}\right)^{\dagger}, \quad \nu=1,2, \cdots,
$$

where $y_{n}, n=1,2, \cdots$ is a scalar associated with the mesh point $x_{n}$, where $x_{n}=$ $(n-1) h$. Let $\mathrm{f}_{\nu}$ be an $s$-vector

$$
\mathbf{f}_{\nu}=\left(f_{\nu s}, \cdots, f_{(\nu-1) s+1}\right)^{\dagger}
$$

where $f_{n}=f\left(x_{n}, y_{n}\right), n=1,2, \cdots$. All of the quantities $y_{n}, f_{n}, n=1,2, \cdots, \mathbf{y}_{\nu}$ and $\mathbf{f}_{\nu}$ will also appear with a superscript $p$, with $f_{n}{ }^{p}=f\left(x_{n}, y_{n}{ }^{p}\right), n=1,2, \cdots, \mathbf{y}_{\nu}{ }^{p}=$ $\left(y_{\nu s, \ldots}^{p}\right)^{\dagger}$, and $\mathrm{f}_{\nu}{ }^{p}=\left(f_{\nu s, \ldots}^{p}, \ldots\right)^{\dagger}, \nu=1,2, \cdots$.

Let $|M|$ denote the determinant of a matrix $M$; let

$$
A_{0}{ }^{*}, A_{\mu}, A_{\mu}{ }^{p}, B_{\mu}, B_{\mu}{ }^{p}, \mu=0, \cdots, k \text { with }\left|A_{0}\right| \neq 0 \text { and }\left|A_{0}{ }^{p}\right| \neq 0
$$

be $s \times s$ matrices, and consider the following explicit system of difference equations for $\mathrm{y}_{\nu+1}^{p}$ and $\mathrm{y}_{\nu}$, where $B_{0}{ }^{p}=0$ :

$$
\begin{aligned}
& A_{0}{ }^{p} \mathbf{y}_{\nu+1}^{p}+A_{1}{ }^{p} \mathbf{y}_{\nu}{ }^{p}+\sum_{\mu=2}^{k} A_{\mu}{ }^{p} \mathbf{y}_{\nu+1-\mu}+h\left(B_{1}{ }^{p}{ }_{\mathbf{f}_{\nu}}{ }^{p}+\sum_{\mu=2}^{k} B_{\mu}{ }^{p} \mathbf{f}_{\nu+1-\mu}\right)=0, \\
& A_{0} \mathbf{y}_{\nu}+A_{0}{ }^{*} \mathbf{y}_{\nu}{ }^{p}+\sum_{\mu=1}^{k} A_{\mu} \mathbf{y}_{\nu-\mu}+h\left(B_{0} \mathbf{f}_{\nu}{ }^{p}+\sum_{\mu=1}^{k} B_{\mu} \mathbf{f}_{\nu-\mu}\right)=0, \\
& \nu=1,2, \cdots \text {. }
\end{aligned}
$$


Let $\mathbf{w}_{\nu}$ denote the $s$-vector whose components are the exact solution of the differential equation at $x=h \nu s, h(\nu s-1), \cdots, h[(\nu-1) s+1]$. Let $\tilde{\mathbf{y}}_{\nu+1}^{p}$ and $\tilde{\mathbf{y}}_{\nu}$ be the solution of the difference equations (2.3) and (2.4), respectively, for vectors $\mathrm{y}_{\nu+1}^{p}$ and $y_{\nu}$, respectively, when all of the remaining $\mathrm{y}_{\mu}$ and $\mathrm{y}_{\mu}{ }^{p}$ are replaced by $\mathbf{w}_{\mu}$.

Let

$$
\overline{\mathbf{y}}_{\nu}=\left(\mathbf{y}_{\nu+1}^{p}, \mathbf{y}_{\nu}\right)^{\dagger}, \quad \overline{\mathbf{w}}_{\nu}=\left(\mathbf{w}_{\nu+1}, \mathbf{w}_{\nu}\right)^{\dagger}, \quad \overline{\mathbf{f}}_{\nu}=\left(\mathbf{f}_{\nu+1}^{p}, f_{\nu}\right)^{\dagger}
$$

be $2 s$-vectors and let

$$
\begin{aligned}
& \bar{A}_{0}=\left(\begin{array}{ll}
A_{0}{ }^{p} & 0 \\
0 & A_{0}
\end{array}\right), \bar{A}_{1}=\left(\begin{array}{ll}
A_{1}{ }^{p} & A_{2}{ }^{p} \\
A_{0}{ }^{*} & A_{1}
\end{array}\right), \bar{A}_{\mu}=\left(\begin{array}{ll}
0 & A_{\mu+1}^{p} \\
0 & A_{\mu}
\end{array}\right) \\
& \bar{B}_{1}=\left(\begin{array}{ll}
B_{1}{ }^{p} & B_{2}{ }^{p} \\
B_{0} & B_{1}
\end{array}\right), \quad \bar{B}_{\mu}=\left(\begin{array}{ll}
0 & B_{\mu+1}^{p} \\
0 & B_{\mu}
\end{array}\right),
\end{aligned}
$$

be $(2 s \times 2 s)$-matrices with $A_{k+1}^{p}=B_{k+1}^{p}=0$.

With this notation the difference equations (2.3) and (2.4) may be written as

$$
\sum_{\mu=0}^{k} \bar{A}_{\mu} \overline{\mathrm{y}}_{\nu-\mu}+h \sum_{\mu=1}^{k} \bar{B}_{\mu} \overline{\mathrm{f}}_{\nu-\mu}=0, \quad(\nu=k+1, k+2, \cdots)
$$

where $|\bar{A}| \neq 0$. Denote by $(\bar{C})_{q, r}, q, r=1, \cdots, 2 s$ the components of a $(2 s \times 2 s)$ matrix $\bar{C}$, by $(\overline{\mathbf{v}})_{r}, r=1, \cdots, 2 s$ those of a $2 s$-vector $\overline{\mathbf{v}}$, and by $(\mathbf{v})_{r}, r=1, \cdots, s$ those of an $s$-vector $\mathbf{v}$. Then, a typical term of (2.7) is

$$
\begin{aligned}
\left(\bar{B}_{\mu} \overline{\mathbf{f}}_{\nu-\mu}\right)_{q}=\sum_{r=1}^{s}\left(\bar{B}_{\mu}\right)_{q, r}\left(\mathbf{f}_{\nu-\mu}^{p}\right)_{r}+\sum_{\substack{r=s+1 \\
\mu}}^{2 s}\left(\bar{B}_{\mu}\right)_{q, r}\left(\mathbf{f}_{\nu-\mu}\right)_{r}, & 1, \cdots, k, \quad q=1, \cdots, 2 s .
\end{aligned}
$$

Since $\left|\bar{A}_{0}\right| \neq 0$, we can for purposes of computation invert $\bar{A}_{0}$ in (2.7). Then the difference equation (2.7) can be solved for each of the $2 s$ components of the vector $\overline{\mathbf{y}}_{\nu}$ simultaneously on $2 s$ arithmetic processors. Each step in the calculation consists of the additions and multiplications required to form the linear combinations displayed in (2.7) as well as the $2 s$ evaluations of $f$, one at each of the components of $\overline{\mathbf{y}}_{\nu}$ and the appropriate value of $x$.

The class of formulas described by (2.7) is thus tailored for parallel computation. We now determine which among them are stable and convergent as well.

Let $\bar{A}(z)$ and $\bar{B}(z)$ be polynomials in the complex variable $z$ with matrix coefficients given by

$$
\bar{A}(z)=\sum_{\mu=0}^{k} \bar{A}_{\mu} z^{k-\mu}, \quad \bar{B}(z)=\sum_{\mu=1}^{k} \bar{B}_{\mu} z^{k-\mu}
$$

Definition. The difference equation (2.7) is consistent if

$$
\bar{A}(1)=0 \text { and } \bar{B}(1)+s \bar{A}^{\prime}(1)=0
$$

where $^{\prime}=d / d z$. The $q$ th row in the difference equation (2.7), with the superscript $p$ deleted wherever it occurs, may be written as

$$
\sum_{r=1}^{2 s} \sum_{\mu=0}^{k}\left(\bar{A}_{\mu}\right)_{q, r}\left(\overline{\mathbf{y}}_{\nu-\mu}\right)_{r}+h \sum_{r=1}^{2 s} \sum_{\mu=1}^{k}\left(\bar{B}_{\mu}\right)_{q, r}\left(\overline{\mathbf{f}}_{\nu-\mu}\right)_{r}=0, \quad q=1,2, \cdots, 2 s .
$$


This represents a linear multistep difference equation of step number $(k+1) s-$ $\varphi(q)-1$ for $y_{n}$ where $\varphi(q)=\min r$ such that $\left(\bar{A}_{0}\right)_{q, r} \neq 0, r=1, \cdots, 2 s$.

Definition. If the difference equation (2.10) is consistent for each $q=1, \cdots, 2 s$, then the difference equation (2.7) is said to be row-wise consistent.

Definition. The polynomial $|\bar{A}(z)|$ satisfies the root condition if all of its roots are in the closed unit disc and those of modulus unity are simple.

Let

$$
\widetilde{\overline{\mathrm{y}}}_{\nu}=\left(\widetilde{\mathrm{y}}_{\nu+1}^{p}, \widetilde{\mathrm{y}}_{\nu}\right)^{\dagger} \text {. }
$$

Then the local truncation error for (2.7) is given by

$$
\widetilde{\mathrm{e}}_{\nu}=\widetilde{\overline{\mathrm{y}}}_{\nu}-\overline{\mathrm{w}}_{\nu} \text {. }
$$

Hypothesis. We assume henceforth that $f(x, y)$ is continuous in $x$ and Lipschitz continuous in $y$ with Lipschitz constant $l$.

Let us confine our arguments to the fixed interval $0 \leq x \leq 1$. In this case we may introduce the modulus of continuity $\xi(\delta)$ of $y^{\prime}(x)$ as follows:

$$
\xi(\delta)=\max \left|y^{\prime}\left(x^{*}\right)-y^{\prime}(x)\right|, \quad\left|x-x^{*}\right| \leq \delta, \quad x, x^{*} \in[0,1] .
$$

For use below we introduce two sets of scalars $\theta_{i}$ and $\theta_{i}{ }^{\prime}, i=-s,-s^{*}+1, \cdots$, $0,1 \cdots$ by means of the following relations

$$
\begin{aligned}
y^{\prime}\left(x_{n-i}\right) & =y^{\prime}\left(x_{n}\right)+\theta_{i} \xi(i h), \\
y\left(x_{n-i}\right) & =y\left(x_{n}\right)+i h\left[y^{\prime}\left(x_{n}\right)+\theta_{i}{ }^{\prime} \xi(i h)\right] .
\end{aligned}
$$

We now state and prove a lemma concerning the size of the local truncation error.

LEMмA. If the difference equation is consistent or row-wise consistent, then

$$
\widetilde{\overline{\mathbf{e}}_{\nu}}=o(h)
$$

as $h$ tends to zero.

Proof. Let $\overline{\mathbf{u}}$ be the $2 s$-vector $(1, \cdots, 1)^{\dagger}$. Then

$$
\begin{aligned}
-\bar{A}_{0} \widetilde{\overline{\mathbf{e}}}_{\nu}= & \sum_{\mu=0}^{k} \bar{A}_{\mu} \overline{\mathbf{w}}_{\nu-\mu}+h \sum_{\mu=1}^{k} \bar{B}_{\mu} \overline{\mathbf{w}}_{\nu-\mu}^{\prime} \\
= & \sum_{\mu=0}^{k} \bar{A}_{\mu} \overline{\mathbf{w}}_{\nu-k}+s h \sum_{\mu=0}^{k}(k-\mu) \bar{A}_{\mu}\left[\overline{\mathbf{w}}_{\nu-k}^{\prime}+\overline{\mathbf{u}} \theta_{(k-\mu) s}^{\prime} \xi((k-\mu) s h)\right] \\
& +h \sum_{\mu=1}^{k} \bar{B}_{\mu}\left[\overline{\mathbf{w}}_{\nu-k}^{\prime}+\mathbf{u} \theta_{(k-\mu) s} \xi((k-\mu) s h)\right] .
\end{aligned}
$$

Then since $\bar{B}_{0}=0$,

$$
-\bar{A}_{0} \widetilde{\overline{\mathbf{e}}}_{\nu}=\sum_{\mu=0}^{k} \bar{A}_{\mu} \overline{\mathbf{w}}_{\nu-k}+h \sum_{\mu=0}^{k}\left[\bar{B}_{\mu}+(k-\mu) s \bar{A}_{\mu}\right] \overline{\mathbf{w}}_{\nu-k}^{\prime}+o(h) .
$$

From this we see that consistency and $\left|\bar{A}_{0}\right| \neq 0$ gives

$$
\widetilde{\mathbf{e}}_{\nu}=o(h) \text {. }
$$

To obtain the same conclusion from row-wise consistency, we proceed as follows.

Let $\left(\overline{\mathbf{w}}_{\nu-k}\right)_{q}$ be the $q$ th component of $\overline{\mathbf{w}}_{\nu-k}$. Then, by Taylor's theorem 
(2.16)

$$
\overline{\mathbf{w}}_{\nu-k}=\left(\overline{\mathbf{w}}_{\nu-k}\right)_{s} \overline{\mathbf{u}}+h\left(\overline{\mathbf{w}}_{\nu-k}^{\prime}\right)_{s}\left(\begin{array}{c}
s-1 \\
\vdots \\
-s
\end{array}\right)+h\left(\begin{array}{c}
(s-1) \theta_{s-1}^{\prime} \xi((s-1) h) \\
\vdots \\
(-s) \theta_{-s}^{\prime} \xi(s h)
\end{array}\right)
$$

$$
\begin{aligned}
\overline{\mathbf{w}}_{\nu-k}^{\prime}= & \left(\overline{\mathbf{w}}_{\nu-k}^{\prime}\right)_{s} \overline{\mathbf{u}}+\left(\begin{array}{c}
\theta_{s-1} \xi((s-1) h) \\
\vdots \\
\theta_{-s} \xi(s h)
\end{array}\right) \\
\overline{\mathbf{w}}_{\nu-k} & =\left(\overline{\mathbf{w}}_{\nu-k}\right)_{2 s} \overline{\mathbf{u}}+h\left(\overline{\mathbf{w}}_{\nu-k}^{\prime}\right)_{2 s}\left(\begin{array}{c}
2 s-1 \\
\vdots \\
0
\end{array}\right) \\
& +h\left(\begin{array}{c}
(2 s-1) \theta_{2 s-1}^{\prime} \xi((2 s-1) h) \\
\vdots \\
0
\end{array}\right),
\end{aligned}
$$

and

$$
\overline{\mathbf{w}}_{\nu-k}^{\prime}=\left(\overline{\mathbf{w}}_{\nu-k}^{\prime}\right)_{2 s} \overline{\mathrm{u}}+\left(\begin{array}{c}
\theta_{2 s-1} \xi((2 s-1) h) \\
\vdots \\
0
\end{array}\right)
$$

Let $\gamma$ be a variable which takes on the values one or two. Inserting (2.16) and (2.17) into (2.15), or (2.18) and (2.19) into (2.15), gives (2.20) with $\gamma=1$ or 2 , respectively.

$$
\begin{aligned}
-\bar{A}_{0} \widetilde{\tilde{\mathbf{e}}_{\nu}}= & \sum_{\mu=0}^{k} \bar{A}_{\mu}\left(\overline{\mathbf{w}}_{\nu-k}\right)_{\gamma s} \overline{\mathbf{u}}+h \sum_{\mu=0}^{k} \bar{A}_{0}\left(\mathbf{w}_{\nu-k}^{\prime}\right)_{\gamma s}\left(\begin{array}{c}
\gamma s-1 \\
\vdots \\
(\gamma-2) s
\end{array}\right) \\
& +h \sum_{\mu=0}^{k}\left[\bar{B}_{\mu}+(k-\mu) s \bar{A}_{\mu}\right]\left(\mathbf{w}_{\nu-k}^{\prime}\right)_{\gamma s} \overline{\mathbf{u}}+o(h) .
\end{aligned}
$$

Then

$$
\begin{aligned}
\left(-\bar{A}_{0} \widetilde{\mathbf{e}}_{\nu}\right)_{q}= & \left(\overline{\mathbf{w}}_{\nu-k}\right)_{\gamma s} \sum_{r=1}^{2 s} \sum_{\mu=0}^{k}\left(\bar{A}_{\mu}\right)_{q, r}+h\left(\overline{\mathbf{w}}_{\nu-k}^{\prime}\right)_{\gamma s} \sum_{r=1}^{2 s} \sum_{\mu=0}^{k}\left(\bar{B}_{\mu}\right)_{q, r} \\
& +h\left(\overline{\mathbf{w}}_{\nu-k}^{\prime}\right)_{\gamma s} \sum_{r=1}^{2 s} \sum_{\mu=0}^{k}[(k-\mu) s+\gamma s-r]\left(\bar{A}_{\mu}\right)_{q, r}+o(h) .
\end{aligned}
$$

Let

$$
\begin{aligned}
\gamma & =1, \quad 1 \leq q \leq s, \\
& =2, \quad s+1 \leq q \leq 2 s .
\end{aligned}
$$

Then an inspection of (2.6) shows that row-wise consistency gives

$$
\left(-\bar{A}_{0} \widetilde{\overline{\mathbf{e}}}_{\nu}\right)_{q}=o(h) .
$$

This and $\left|\bar{A}_{0}\right| \neq 0$ implies the lemma.

We now state and prove an auxiliary lemma dealing with polynomials which 
obey the root condition.

Lemma. Let the polynomial $|M(z)|=\left|\sum_{\mu=0}^{k} M_{\mu} z^{k-\mu}\right|,\left|M_{0}\right| \neq 0$, where the $M_{\mu}$ are matrices, obey the root condition. Then the matrix $\left[z^{k} M(1 / z)\right]^{-1}$ is analytic in a neighborhood of $z=0$. If $M_{\mu}, \mu=0,1, \cdots$ are matrices given by

$$
\left[z^{k} M\left(\frac{1}{z}\right)\right]^{-1}=\sum_{\mu=0}^{\infty} M_{\mu} z^{\mu},
$$

then

$$
\max _{\mu}\left\|M_{\mu}\right\|=M<\infty .
$$

Proof. Since $z^{k} M(1 / z)=\sum_{\mu=0}^{k} M_{\mu} z^{\mu}$ and $\left|M_{0}\right| \neq 0$, it is clear that $\left[z^{k} M(1 / z)\right]^{-1}$ is analytic in a neighborhood of the origin. Since $\left|z^{k} M(1 / z)\right|=z^{2 s k}|M(1 / z)|$, the root condition locates the roots of the polynomial $\left|z^{k} M(1 / z)\right|$ outside the open unit disc, and those on the unit disc are simple. Since

$$
\left.\left[z^{k} M(1 / z)\right]^{-1}=\text { [matrix of polynomials }\right] /\left|z^{k} M(1 / z)\right|,
$$

it suffices to show that the power series for the reciprocal polynomial $\left|z^{k} M(1 / z)\right|^{-1}$ has bounded coefficients. ${ }^{1}$ We omit the proof of this fact since it consists of a straightforward computation.

Now let us define the accumulated truncation error

$$
\overline{\mathbf{e}}_{\nu}=\overline{\mathbf{y}}_{\nu}-\overline{\mathbf{w}}_{\nu} \text {. }
$$

We can state and prove the following theorem.

Thеовем. Let the difference equation (2.7) be consistent or row-wise consistent and let it be solved with compatible initial data; i.e., with $\overline{\mathbf{e}}_{v}=\mathbf{y}_{\nu}-\overline{\mathbf{w}}_{\nu}$, where

$$
\overline{\mathbf{e}}_{\nu}=o(1), \quad \nu=1, \cdots, k, \quad \text { as } h \rightarrow 0 .
$$

If $|\bar{A}(z)|$ satisfies the root condition, then

$$
\overline{\mathbf{e}}_{\nu}=o(1), \quad \nu=1, \cdots,\{1 / s h\} .
$$

Here, the last symbol denotes the integer part of $1 /$ sh.

Proof. With the two lemmas just established, the proof follows along the lines of the scalar case as in [4]. We omit the details.

Remark. The notion of row-wise consistency which we have introduced here and the use of the root condition on the determinant $|\bar{A}(z)|$ are the two ingredients of the convergence theorem proved in this section. These are not the only hypotheses relating to consistency and stability which could be made. However, we have found these hypotheses to be particularly useful for constructing systems of parallel integration methods. This is demonstrated in the following section. The theorem given here is sufficient but not necessary for convergence. For example, the determinant of $A(z)=I z-I$ has a multiple root at $z=1$, but, according to a theorem of B. Dejon [1], this matrix corresponds to a stable method.

3. Classes of Stable and Consistent Parallel Methods. In this section we will derive sets of parallel integration formulas. We start with the general finite-difference

${ }^{1}$ We are indebted to A. Hoffman for this observation. 
equation (2.7) and make some specializing assumptions. These assumptions enable us to obtain classes of systems which are row-wise consistent and whose associated matrices have determinants which obey the root condition. In the cases $s=1$ and 2 of two and four processors, respectively, explicit cases of these integration formulas are exhibited.

A. The Two Processor Case $(s=1)$. Consider a combination of a single predictor and a single corrector formula which form a system of a generalized Adams type; i.e., for which

$$
\bar{A}(z)=\bar{A}_{0} z^{k}+\bar{A}_{1} z^{k-1} ; \quad \bar{A}_{j}=0, \quad(2 \leq j \leq k) .
$$

Recall that since $s=1$, the $\bar{A}_{j}, j=0,1, \cdots$, are $2 \times 2$ matrices, and the $A_{j}$ and $B_{j}$ are scalars, denoted by $a_{j}$ and $b_{j}$, respectively. Condition (3.1) implies $a_{j}=0(2 \leq j$ $\leq k)$, and $a_{j}{ }^{p}=0(3 \leq j \leq k)$. As a consequence, the two integration formulas have the structure

$$
\begin{aligned}
a_{0}{ }^{p} y_{n+1}^{p}+a_{1}^{p} y_{n}{ }^{p}+a_{2}{ }^{p} y_{n-1}+h\left(b_{1}{ }^{p} f_{n}{ }^{p}+b_{2}{ }^{p} f_{n-1}+\cdots\right) & =0 \\
a_{0} y_{n}+a_{0}{ }^{*} y_{n}{ }^{p}+a_{1} y_{n-1}+h\left(b_{0} f_{n}{ }^{p}+b_{1} f_{n-1}+\cdots\right) & =0 .
\end{aligned}
$$

For formulas of this type, $|\bar{A}(z)|=z^{2(k-1)}\left|\left(\bar{A}_{0} z+\bar{A}_{1}\right)\right|$. Thus, $z=0$ is a root of multiplicity at least $2(k-1)$. The two remaining roots of $|\bar{A}(z)|=0$ are the solutions of the quadratic equation $\left|\bar{A}_{0} z+\bar{A}_{1}\right|=0$ or

$$
a_{0} a_{0}^{p} z^{2}+\left(a_{0}^{p} a_{1}+a_{0} a_{1}^{p}\right) z+\left(a_{1} a_{1}^{p}-a_{0}^{*} a_{2}^{p}\right)=0 .
$$

A necessary condition for row-wise consistency is

$$
a_{2}^{p}=-\left(a_{0}^{p}+a_{1}^{p}\right) ; \quad a_{0}^{*}=-\left(a_{0}+a_{1}\right) .
$$

If we substitute in (3.3) from (3.4), we find that the solutions of (3.3) are $z_{1}=1$, and $z_{2}=\left[1+\left(a_{1} / a_{0}\right)+\left(a_{1}^{p} / a_{0}^{p}\right)\right]$. Without loss of generality we may normalize the rows in such a way that $a_{0}^{p}=a_{0}=-1$. Then $z_{2}=-1+\left(a_{1}+a_{1}^{p}\right), a_{2}^{p}=1-a_{1}^{p}$, and $a_{0}^{*}=1-a_{1}$. If we let

$$
a_{1}=1-a_{1}^{p}=a_{2}^{p},
$$

in which case $a_{0}{ }^{*}=a_{1}{ }^{p}$, then $z_{2}=0$, and the root condition is guaranteed. More generally, $-1 \leq z_{2}<1$ if and only if

$$
-a_{1}^{p} \leq a_{1}<2-a_{1}^{p}
$$

The value $z_{2}=1$ must be excluded in order to avoid a double root of $z=1$. The freedom in choosing $a_{1}$ within the limits imposed by (3.6) might be used for accuracy purposes.

For simplicity assume finally that ${a_{1}}^{p}=0$. In this case $a_{1}=a_{2}{ }^{p}=1$ and $a_{0}{ }^{*}=0$ if (3.5) is imposed or $0 \leq a_{1}<2$ if the less stringent condition (3.6) is used. If we incorporate (3.5) and the other conditions imposed above into (3.2), we get

$$
\begin{aligned}
-y_{n+1}^{p}+y_{n-1}+h\left(b_{1}{ }^{p} f_{n}{ }^{p}+\sum_{j=2}^{r+1} b_{j}{ }^{p} f_{n-j+1}\right) & =0, \\
-y_{n}+y_{n-1}+h\left(b_{0} f_{n}{ }^{p}+{ }_{j=1}^{r} b_{j} f_{n-j}\right) & =0 .
\end{aligned}
$$


If the $b^{p}$ 's and $b$ 's are chosen in such a way that the order of row-wise accuracy is $\geq 1$, then we are assured of row-wise consistency. Notice that, if no distinction is made between $y$ and $y^{p}$, the second formula by itself is a closed Adams formula. The coefficients $b_{j}{ }^{p}$, can be determined in the usual manner. Stability is guaranteed by condition (3.5), and thus convergence according to the main theorem of $\$ 2$.

If the upper limits of summation over the $f$ 's in the predictor and corrector formulas are as indicated in (3.7), and if, for a given $r$, the $b$ 's and $b^{p}$ 's are chosen in such a way that the individual formulas are of maximum order of accuracy, that order is the same for both formulas $(=r+1)$. As an example, the second-order formulas of type (3.7) (in which $b_{2}{ }^{p}$ happens to vanish) are

$$
\begin{aligned}
y_{n+1}^{p} & =y_{n-1}+2 h f_{n}{ }^{p}, \\
y_{n} & =y_{n-1}+\frac{h}{2}\left(f_{n}{ }^{p}+f_{n-1}\right) .
\end{aligned}
$$

The corresponding pairs of formulas of order 3 and 4 are

$$
\begin{aligned}
y_{n+1}^{p} & =y_{n-1}+\frac{h}{3}\left(7 f_{n}{ }^{p}-2 f_{n-1}+f_{n-2}\right), \\
y_{n} & =y_{n-1}+\frac{h}{12}\left(5 f_{n}^{p}+8 f_{n-1}-f_{n-2}\right),
\end{aligned}
$$

and

$$
\begin{aligned}
y_{n+1}^{p} & =y_{n-1}+\frac{h}{3}\left(8 f_{n}{ }^{p}-5 f_{n-1}+4 f_{n-2}-f_{n-3}\right), \\
y_{n} & =y_{n-1}+\frac{h}{24}\left(9 f_{n}{ }^{p}+19 f_{n-1}-5 f_{n-2}+f_{n-3}\right),
\end{aligned}
$$

respectively.

B. The Case of Four or More Processors. For four or more machines $(s \geq 2)$, the coefficients of the predictor-corrector formulas (2.3) and (2.4) are $s \times s$ matrices. Assume again that $\bar{A}_{j}=0$ for $2 \leq \mu \leq k$, or that $A_{\mu}=0$ for $2 \leq \mu \leq k$, and $A_{\mu}{ }^{p}=$ 0 for $3 \leq \mu \leq k$. Then $|\bar{A}(z)|=z^{2 s(k-1)}\left|\bar{A}_{0} z+\bar{A}_{1}\right|$ has a root $z=0$ of multiplicity at least $2 s(k-1)$. The remaining $2 s$ roots are those of $\left|\bar{A}_{0} z+\bar{A}_{1}\right|$. If $\bar{A}_{0}=-I_{2 s \times 2 s}$ so that $A_{0}{ }^{p}=A_{0}=-I_{s \times s}$, then

$$
\bar{A}_{0} z+\bar{A}_{1}=-\left(\begin{array}{cc}
z I_{s \times s}-A_{1}{ }^{p} & -A_{2}{ }^{p} \\
-A_{0}{ }^{*} & z I_{s \times s}-A_{1}
\end{array}\right) .
$$

Let $A_{0}{ }^{*}=0$, and let $A_{1}{ }^{p}$ and $A_{1}$ have the structure

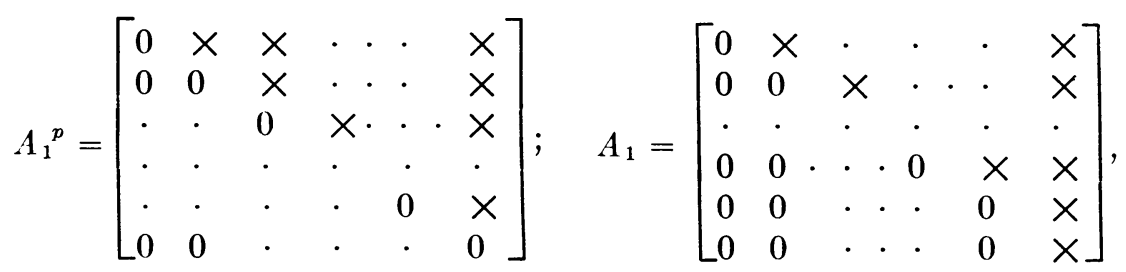


where crosses represent entries that need not vanish. For $\bar{A}_{0}=-I_{2 s \times 2 s}$ it follows, from (3.12) and from the consistency requirement for the last row of the corrector formula, that the lower right-hand corner element of $A_{1}$ is unity. The indicated structure of $A_{1}{ }^{p}$ and $A_{1}$ gives $\left|\bar{A}_{0} z+\bar{A}_{1}\right|=-z^{2 s-1}(z-1)$ and thus $|\bar{A}(z)|$ has a root $z=0$ of multiplicity $(2 s k-1)$ and the single (principle) root $z=1$. Thus $|\bar{A}(z)|$ satisfies the root condition. Row-wise consistency is guaranteed if the coefficients are chosen in such a way that the order of accuracy of the formulas represented by the individual rows upon identifying $y^{p}$ and $y$ is $\geq 1$. The most general set of formulas satisfying all the conditions imposed above is of the form

$$
\begin{array}{r}
-y_{(\nu+1) s-q+1}^{p}+\sum_{r=q+1}^{s}\left(A_{1}^{p}\right)_{q, r} y_{\nu s-r+1}^{p}+\sum_{r=1}^{s}\left(A_{2}^{p}\right)_{q, r} y_{(\nu-1) s-r+1}+h(\cdots)=0, \\
-y_{\nu s-q+1}+\sum_{r=q+1}^{s}\left(A_{1}\right)_{q, r} y_{(\nu-1) s-r+1}+h(\cdots)=0, \\
-y_{(\nu-1) s+1}+y_{(\nu-2) s+1}+h(\cdots)=0, \quad(q=1,2, \cdots, s-1),
\end{array}
$$

They can be thought of as obtained from formulas

$$
\begin{aligned}
& -y_{(\nu+1) s-q+1}+\sum_{r=q+1}^{s}\left(A_{1}^{p}\right)_{q, r} y_{\nu s-r+1} \\
& +\sum_{r=1}^{s}\left(A_{2}^{p}\right)_{q, r} y_{(\nu-1) s-r+1}+h \sum_{j=1}\left(C^{p}\right)_{q, j} f_{\nu s-j+1}=0, \\
& \left.-y_{\nu s-q+1}+\sum_{r=q+1}^{s}\left(A_{1}\right)_{q, r} y_{(\nu-1) s-r+1}+h \sum_{j=1}(C)_{q, j} f_{\nu s-j+1}=0,2, \cdots, s\right), \\
& -y_{(\nu-1) s+1}+y_{(\nu-2) s+1}+h \sum_{j=1}(C)_{s, j} f_{\nu s-j+1}=0,
\end{aligned}
$$

respectively. Here, $C$ and $C^{p}$ are rectangular matrices of $s$ rows and as many columns as are needed; the elements of $C$ and $C^{p}$ are those of the $B_{\mu}$ 's and $B_{\mu}{ }^{p}$ 's taken in appropriate order. The upper limits of the summations over the $f$ 's in (3.15) and (3.16) are related to the row-wise order of accuracy and, in general, depend on $q$.

The formulas considered above are particularly simple if we let $A_{1}{ }^{p}=0$. Methods (3.17) and (3.18) listed below are of this type with $s=2$. In addition, they have the properties $\left(A_{2}^{p}\right)_{1,2}=\left(A_{2}^{p}\right)_{2,2}=C_{2,1}=0$, which are discussed in $\$ 4$. Within each method, the order of accuracy is the same for all rows and is $O(h)$ and $O\left(h^{2}\right)$, respectively, for the two methods:

$$
\begin{aligned}
y_{2 n+2}^{p} & =y_{2 n-2}+4 h f_{2 n}^{p}, \\
y_{2 n+1}^{p} & =y_{2 n-2}+3 h f_{2 n}^{p}, \\
y_{2 n} & =y_{2 n-3}+3 h f_{2 n}^{p}, \\
y_{2 n-1} & =y_{2 n-3}+2 h f_{2 n-1}^{p},
\end{aligned}
$$




$$
\begin{aligned}
y_{2 n+2}^{p} & =y_{2 n-2}+4 h f_{2 n}^{p}, \\
y_{2 n+1}^{p} & =y_{2 n-2}+\frac{3 h}{2}\left(f_{2 n}^{p}+f_{2 n-1}^{p}\right), \\
y_{2 n} & =y_{2 n-3}-\frac{h}{2}\left(3 f_{2 n}^{p}-9 f_{2 n-1}^{p}\right), \\
y_{2 n-1} & =y_{2 n-3}+2 h f_{2 n-2} .
\end{aligned}
$$

4. Discussion and Numerical Results. Open linear multistep methods have the advantage that they require a minimal amount of work per integration step (one single evaluation of $f(x, y)$ ). However, their asymptotic stability is strongly restricted, and they force us to use a relatively small $h$. Therefore, the over-all amount of work in carrying the integration over a given interval may be large, nevertheless. Closed formulas are more stable and permit the use of larger $h$ 's but, in general, require the solution of functional equations at each time step to obtain the new value of the dependent variable. A predictor-corrector (PC)-scheme appears to give a good compromise between the two types of formulas. Although the amount of work per step is larger than for the open formula (at least two evaluations of $f(x, y)$ ), a PC-scheme is considered advantageous from an over-all point of view since it is normally much more stable and, with the same step number, somewhat more accurate than an open formula.

If a parallel PC-method for $2 s$ processors gives the same accuracy as a sequential scheme with the same step size, it is $2 s$ times as fast. If the parallel scheme is less accurate than the sequential one, it must be used with a step, $h_{p}$, which is smaller than the step $h_{s}$ used in the sequential scheme in order for the accumulated error to be the same; i.e., $h_{p}=\rho h_{s}, \rho<1$. The quantity $2 s \rho$, which we henceforth denote as $\Phi$, is called the speed increase factor for the parallel method. $\Phi$ is the number of times faster a parallel scheme is, compared with a sequential scheme of the same accuracy. Clearly, a parallel scheme loses its usefulness if $\Phi \ngtr 1$.

Methods (3.8), (3.9), and (3.10), to be referred to as $\mathrm{P} 1 \omega, \omega=2,3$, 4, respectively, where the digit 1 indicates the value of $s$ and $\omega$ is the row-wise order of accuracy, have been tested numerically with equation

$$
y^{\prime}=-y-w \pi e^{-x} \sin w \pi x, \quad y(0)=1+r,
$$

which has the exact solution

$$
y(x)=e^{-x}(r+\cos w \pi x) .
$$

The calculation was done from $x_{0}=0$ through $x_{n}=1$, with $h=n^{-1}$, and $n=24$, 48,96 , and 192. The accumulated truncation error was printed out at various values of $x$ (the influence of the round-off errors was largely eliminated by using double precision arithmetic). With $r=0$, the error at $x=1$ is plotted as a function of $h$ in doubly logarithmic fashion, for $w=0$ in Fig. 1, and for $w=6$ in Fig. 2. The results are compared to those obtained by serial predictor-corrector methods of corresponding order (referred to as $\mathrm{S} 1 \omega, \omega=2,3,4$ ) ) i.e., by the Adams pairs

$$
y_{n+1}^{p}=y_{n}+\frac{h}{2}\left(3 f_{n}-f_{n-1}\right), y_{n+1}=y_{n}+\frac{h}{2}\left(f_{n+1}^{p}+f_{n}\right),
$$




$$
\begin{aligned}
& y_{n+1}^{p}=y_{n}+\frac{h}{12}\left(23 f_{n}-16 f_{n-1}+5 f_{n-2}\right), \\
& y_{n+1}=y_{n}+\frac{h}{12}\left(5 f_{n+1}^{p}+8 f_{n}-f_{n-1}\right),
\end{aligned}
$$

and

$$
\begin{aligned}
& y_{n+1}^{p}=y_{n}+\frac{h}{24}\left(55 f_{n}-59 f_{n-1}+37 f_{n-2}-9 f_{n-3}\right) \\
& y_{n+1}=y_{n}+\frac{h}{24}\left(9 f_{n+1}^{p}+19 f_{n}-5 f_{n-1}+f_{n-2}\right)
\end{aligned}
$$

respectively. The limits of usefulness at which $\Phi=1$; i.e., $\rho=\frac{1}{2}$, are identified by $\mathrm{L} 1 \omega, \omega=2,3,4$.

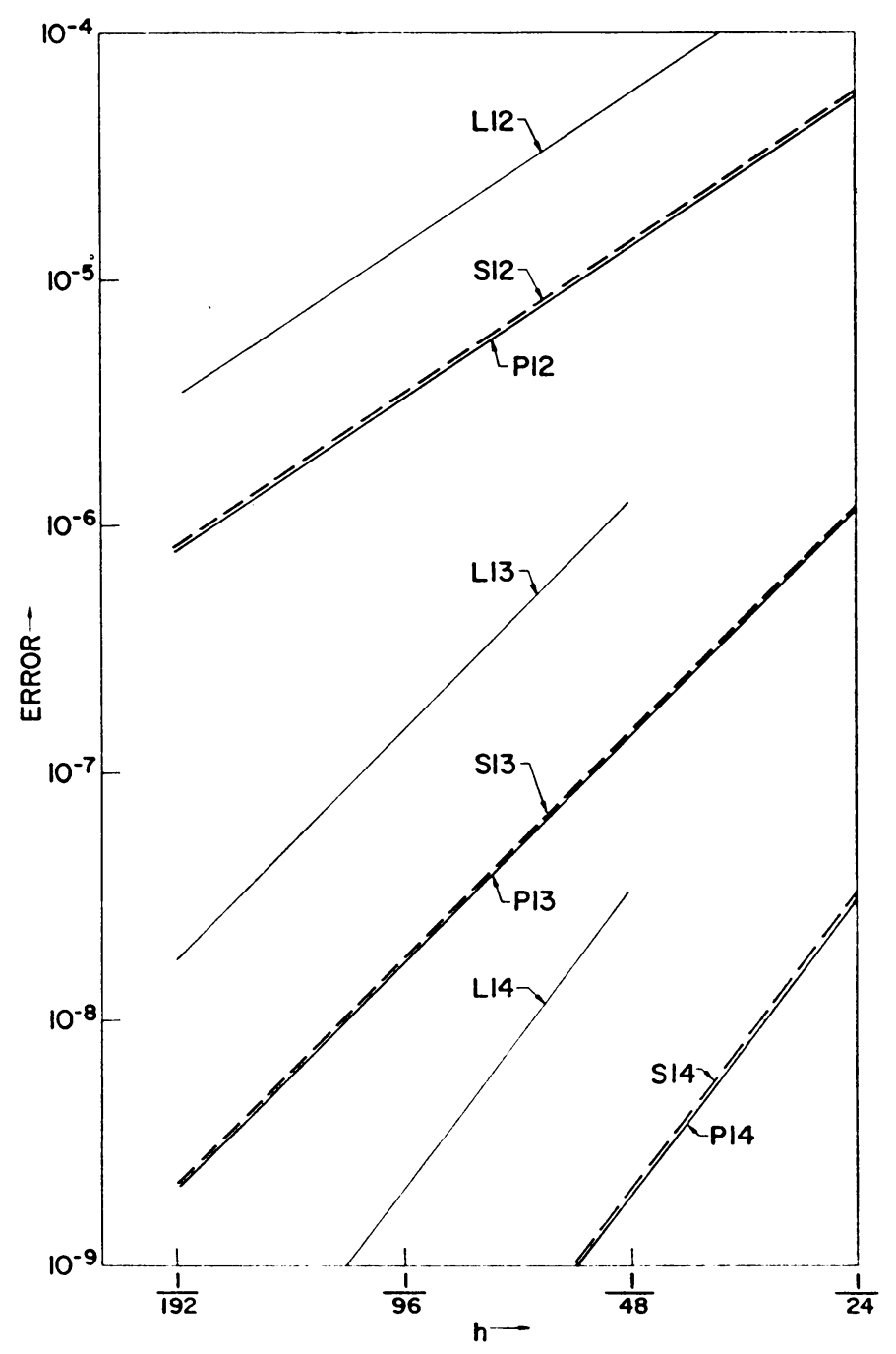

Figure 1. Two processors. Nonoscillatory case 
We can observe a rising sensitivity to $h$ for increasing $\omega$ (i.e., an increasing slope of the curves). Clearly, this effect is even more dramatic as far as the frequency $w$ is concerned. While for $w=0$, there is a considerable improvement in accuracy as $\omega$ increases, the lowest-order methods are the best for $w=6$, except for very small $h$. This exhibits the well-known fact that low-order formulas may perform better with very irregular data than high-order ones, except for extremely small values of $h$. Steps of such a small size would be undesirable for practical purposes.

In practically all cases we have $\Phi=2$. It is true that, for $w=0$, the increase in speed obtained in using P12 instead of S12 can be destroyed by replacing S12 by $\mathrm{S} 13$, since S13 gives the same accuracy as P12 with an $h$ more than twice as big as that used in P12. But then again P13 beats $\mathrm{S} 13$ with $\Phi=2$, etc. For $w=6$ it seems impossible to beat the performance of a given parallel method by a serial method of higher order except possibly for steps which are much smaller than those used in the figures.

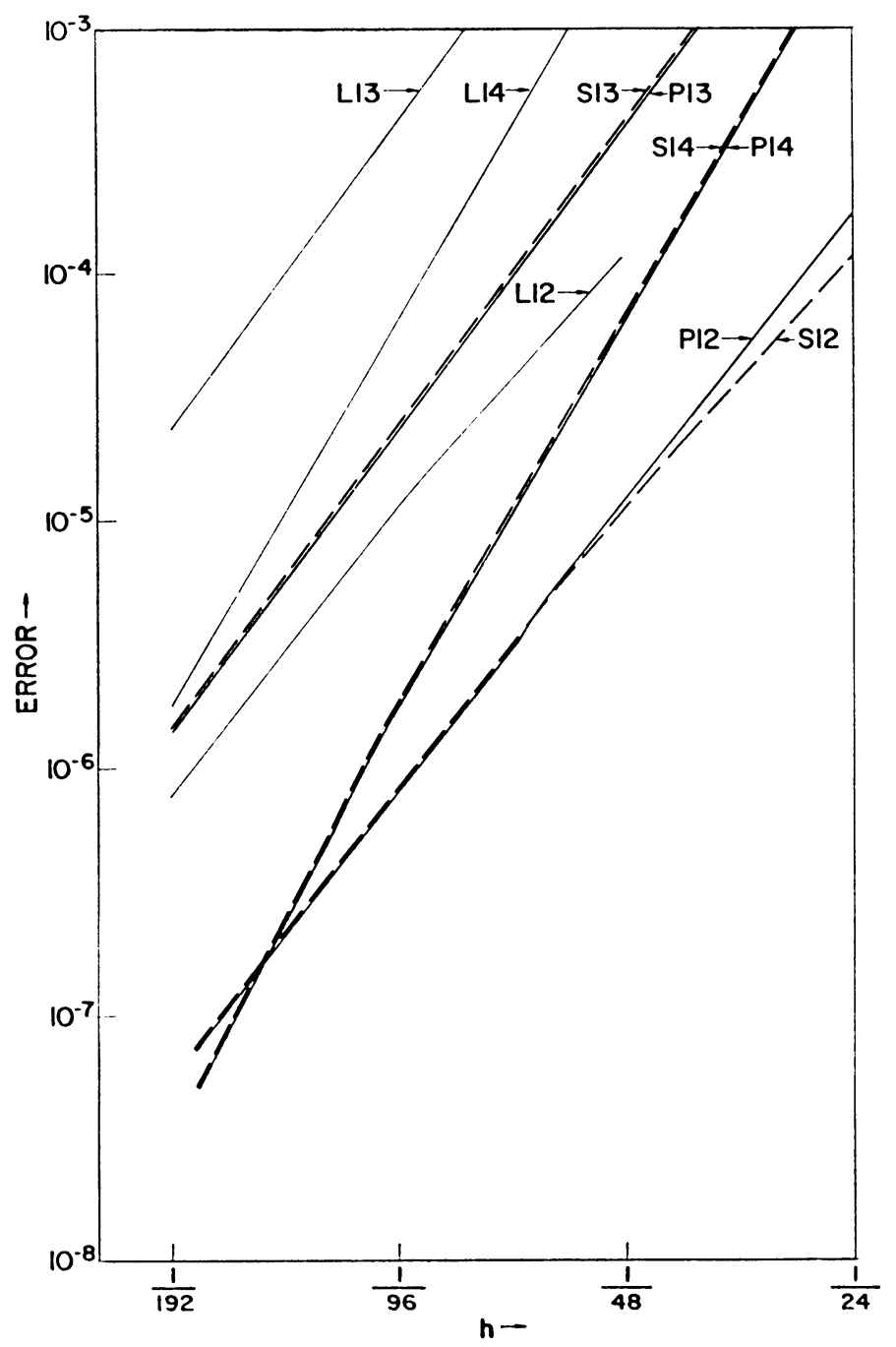

Figure 2. Two processors. High frequency case 
As examples of methods for four processors, formulas (3.17) and (3.18) (referred to as $\mathrm{P} 2 \omega, \omega=1,2$, respectively, in accordance with the code used in the previous subsection), have been applied to (4.4) as in the two-processor case. The results, as well as the limits of usefulness, $L 2 \omega$, corresponding to $\Phi=1$ or $\rho=\frac{1}{4}$, are plotted, in Fig. 3 for $w=0$, and in Fig. 4 for $w=6$, and compared to the sequential Adams PC pairs,

$$
y_{n+1}^{p}=y_{n}+h f_{n}, \quad y_{n+1}=y_{n}+h f_{n+1}^{p},
$$

and (4.6), i.e., $\mathrm{S} 1 \omega, \omega=1,2$, respectively. For $w=0$ we have $\Phi \approx 2$ when $\omega=1$, and $\Phi>4$ when $\omega=2$. Thus, P22 is much more accurate than S12 with the same step size, and for that reason it was unnecessary to plot L22. For $w=6$, we found $\Phi \approx 4$ with $\omega=1$, and $\Phi \approx 3$ with $\omega=2$.

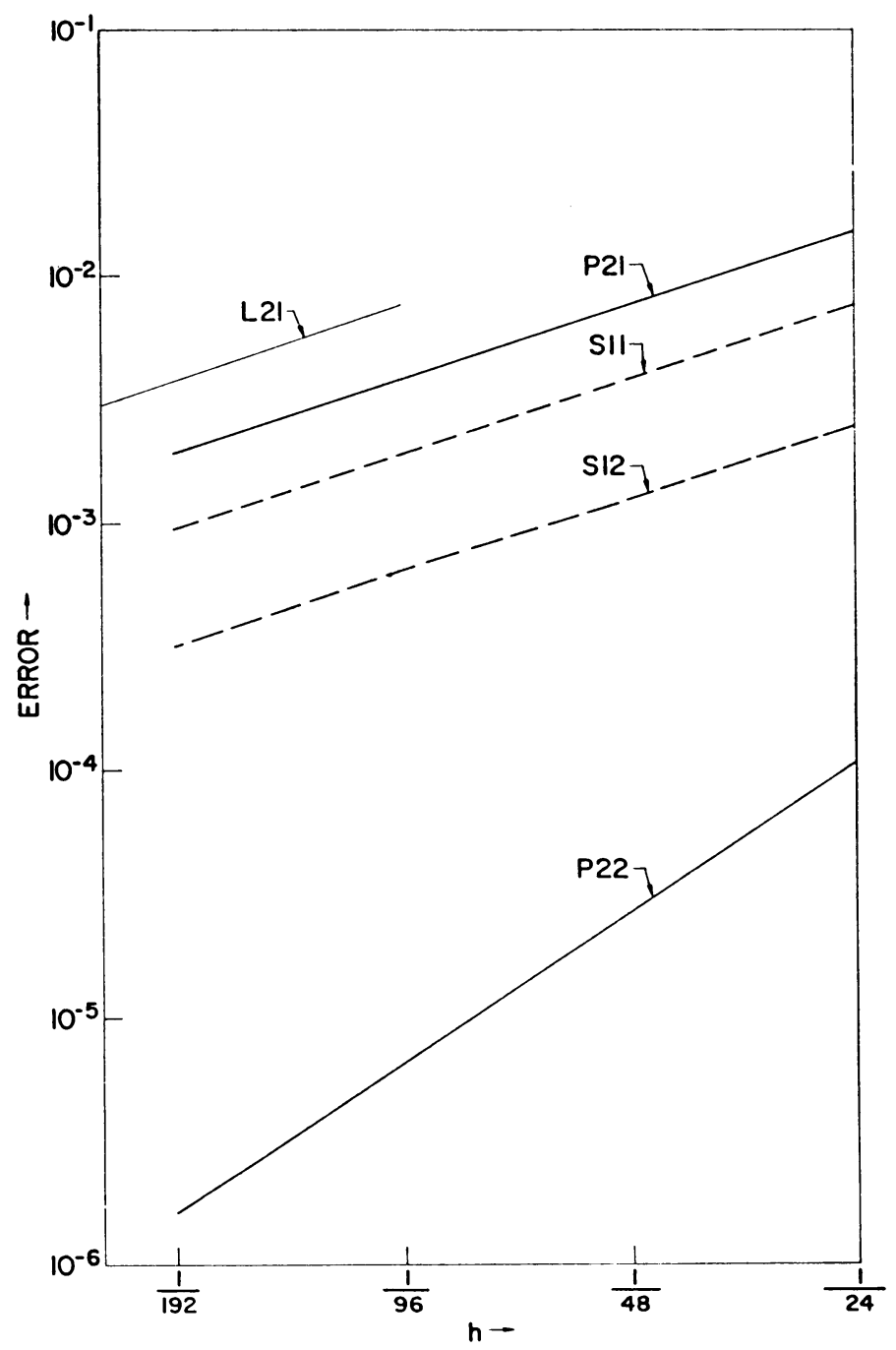

Figure 3. Four processors. Nonoscillatory case 
Appendix.

Parallel Second-and Third-Order Runge-Kutta Formulas. Parallel second- and third-order Runge-Kutta formulas have been derived by a modification of Kopal's [5] approach. In a standard serial Runge-Kutta method of order $\omega$, the integration step leading from $y_{0}$ to $y_{1}$ consists of computing substitutions,

$$
\begin{aligned}
& k_{1}=h f\left(x_{0}, y_{0}\right) \\
& k_{n}=h f\left(x_{0}+\alpha_{n} h, y_{0}+\sum_{j=1}^{n-1} \beta_{n, j} k_{j}\right), \quad(n=2, \cdots, \omega)
\end{aligned}
$$

and then forming a linear combination,

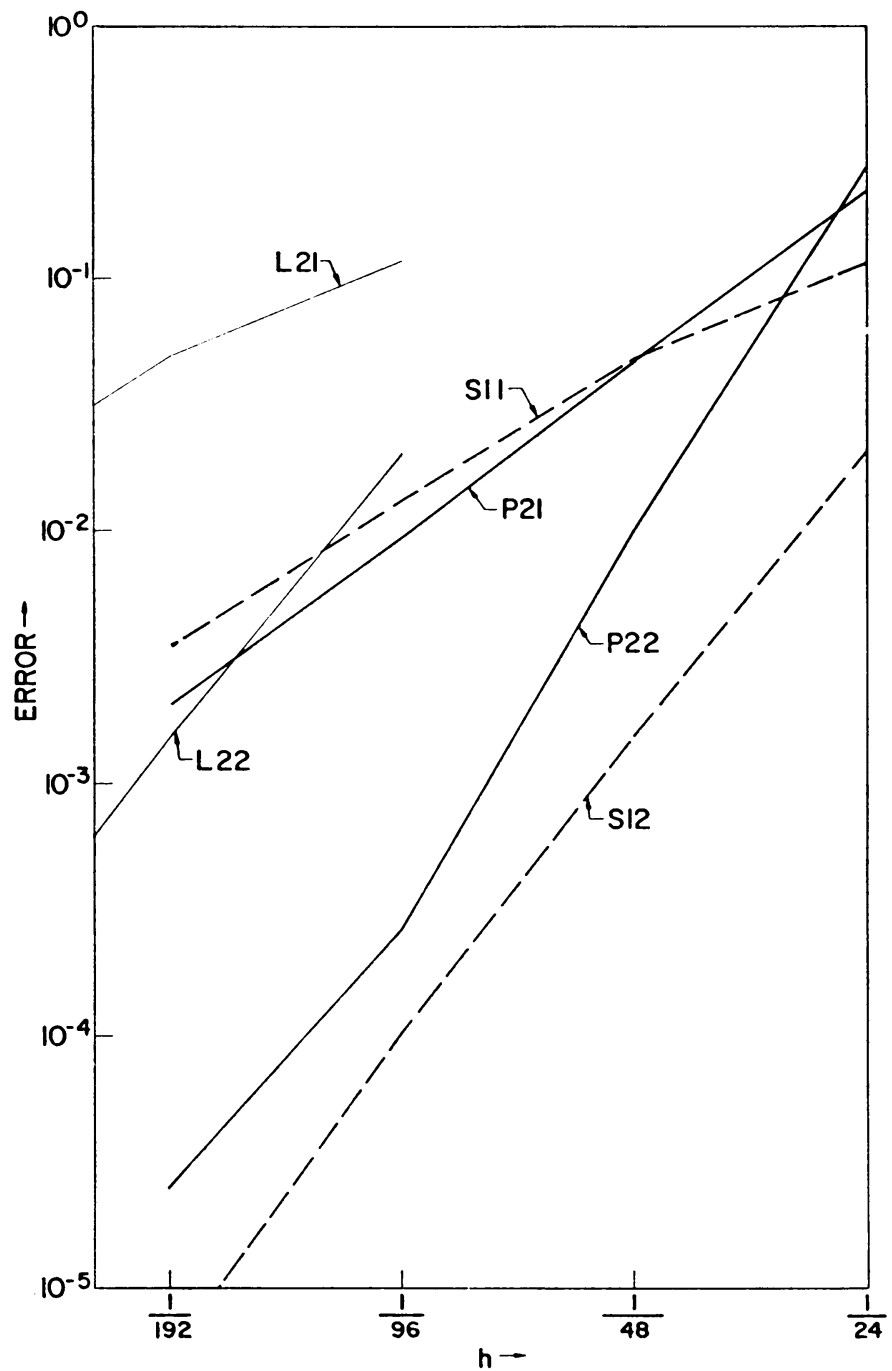

FIGURe 4. Four processors. High frequency case 


$$
y_{1}=y_{0}+\sum_{j=1}^{\omega} R_{j} k_{j},
$$

with appropriate values of $\alpha_{n}, \beta_{n, j}$, and $R_{n}$.

In the parallel computation of a third-order approximation $y_{1}{ }^{(3)}$, first-and secondorder approximations, $y_{0}{ }^{(1)}$ and $y_{0}{ }^{(2)}$, respectively in addition to $y_{0}{ }^{(3)}$, are involved. Thus in order to continue the calculation, $y_{1}{ }^{(1)}$ and $y_{1}{ }^{(2)}$ as well as $y_{1}{ }^{(3)}$ have to be computed. As a consequence, the third-order parallel method gives a second-order parallel scheme as a by-product. For the reasons explained below the formulas of the parallel schemes have the structure:

$$
\begin{aligned}
k_{1} & =h f\left(x_{0}, y_{0}{ }^{(1)}\right), \\
y_{1}{ }^{(1)} & =y_{0}{ }^{(1)}+k_{1}
\end{aligned}
$$

for the first-order approximation. This is identical with the standard serial first-order case.

$$
\begin{aligned}
k_{1}{ }^{(2)} & =k_{1}\left(=h f\left(x_{0}, y_{0}{ }^{(1)}\right)\right), \\
k_{2} & =h f\left(x_{0}+\alpha h, y_{0}{ }^{(1)}+\beta k_{1}{ }^{(2)}\right), \\
y_{1}{ }^{(2)} & =R_{1}{ }^{(2)} k_{1}{ }^{(2)}+{R_{2}}^{(2)} k_{2},
\end{aligned}
$$

for the second-order approximation.

$$
\begin{aligned}
k_{1}{ }^{(3)} & =k_{1}, \\
k_{2}{ }^{(3)} & =k_{2}, \\
k_{3} & =h f\left(x_{0}+\alpha_{1} h, y_{0}{ }^{(2)}+\beta_{1} k_{1}{ }^{(3)}+\gamma_{1} k_{2}{ }^{(3)}\right), \\
y_{1}{ }^{(3)} & =R_{1}{ }^{(3)} k_{1}{ }^{(3)}+{R_{2}}^{(3)} k_{2}{ }^{(3)}+R_{3}{ }^{(3)} k_{3},
\end{aligned}
$$

for the third-order approximation.

Formula (A.3) needs no explanation. In formula (A.4) $k_{1}$ and $k_{2}$ are computed in terms of $y_{0}{ }^{(1)}$ which is accurate to $O(h)$ instead of $y_{0}{ }^{(2)}$ which is accurate to $O\left(h^{2}\right)$ and which would be used in a serial second-order method. Nevertheless, $k_{1}$ and $k_{2}$ are accurate to $O\left(h^{2}\right)$ since the $f^{\prime}$ 's are multiplied by $h$. According to Kopal, $y_{1}{ }^{(2)}$ given by the last of equations (A.4) is $O\left(h^{2}\right)$ if $R_{1}{ }^{(2)}=1-(2 \alpha)^{-1}, R_{2}{ }^{(2)}=(2 \alpha)^{-1}$, and $\beta=\alpha$, where $\alpha$ is a parameter at our disposal.

In (A.5), $k_{3}$ is computed from a $y_{0}^{(2)}$ and $k_{1}$ and $k_{2}$, all of which are $O\left(h^{2}\right)$. As a consequence, $k_{3}$ is $O\left(h^{3}\right)$ for the reason indicated above. In order that $y_{1}{ }^{(3)}$ be accurate to $O\left(h^{3}\right)$ in this parallel mode, i.e., in order for the $O\left(h^{2}\right)$ terms to drop out, the first of equations (IV-L-34) of Kopal must be split into $R_{1}{ }^{(3)}+R_{2}{ }^{(3)}=0, R_{3}{ }^{(3)}=1$. With the following choice for $\alpha$

$$
\alpha=2\left(1-3 \alpha_{1}^{2}\right)\left[3\left(1-2 \alpha_{1}\right)\right]^{-1},
$$

there is a one-parameter family of solutions: $R_{1}{ }^{(3)}=\left(2 \alpha_{1}-1\right)(2 \alpha)^{-1}, R_{2}{ }^{(3)}=$ $\left(1-2 \alpha_{1}\right)(2 \alpha)^{-1}, R_{3}{ }^{(3)}=1, \gamma_{1}=(6 \alpha)^{-1}$, and $\beta_{1}=\alpha_{1}-(6 \alpha)^{-1}$. If in particular we let $R_{1}{ }^{(3)}=R_{3}{ }^{\left({ }^{3)}\right.}$ for symmetry as suggested by Kopal, then $R_{1}{ }^{(3)}=1, R_{2}{ }^{(3)}=-1, R_{3}{ }^{(3)}$ $=1, R_{1}^{(2)}=-5, R_{2}^{(2)}=6, \alpha=\beta=1 / 12, \alpha_{1}=-7 / 12, \beta_{1}=17 / 12$, and $\gamma_{1}=2$. Coefficients of more similar magnitude are provided by the solution: $R_{1}{ }^{(3)}=$ 
$(2 \sqrt{ } 3)^{-1}, R_{2}^{(3)}=-(2 \sqrt{ } 3)^{-1}, R_{3}^{(3)}=1, R_{1}{ }^{(2)}=R_{2}{ }^{(2)}=2^{-1}, \alpha=\beta=1, \alpha_{1}=2^{-1}$ $+(2 \sqrt{ } 3)^{-1}, \beta_{1}=3^{-1}+(2 \sqrt{ } 3)^{-1}$, and $\gamma_{1}=1 / 6$.

The parallel character of the present formulas results from the fact that any two methods, represented by formulas (A.i) and (A.j) respectively, where $i \neq j, i, j=3$, 4 , or 5 , have the property that (A.i) is uncoupled from (A.j), but not vice versa, if and only if $i<j$. If we let (A.4) and (A.3) run ahead of (A.5) by one and two steps, respectively, we get (A.7)

$$
\begin{aligned}
k_{1, n+2} & =h f\left(x_{n+2}, y_{n+2}^{(1)}\right), \\
y_{n+3}^{(1)} & =y_{n+2}^{(1)}+k_{1, n+2}, \\
k_{2, n+1} & =h f\left(x_{n+1}+\alpha h, y_{n+1}^{(1)}+\alpha k_{1, n+1}\right), \\
y_{n+2}^{(2)} & =y_{n+1}^{(2)}+\left(1-\frac{1}{2 \alpha}\right) k_{1, n+1}+\frac{1}{2 \alpha} k_{2, n+1}, \\
k_{3, n} & =h f\left(x_{n}+\alpha_{1} h, y_{n}^{(2)}+\left(\alpha_{1}-\frac{1}{6 \alpha}\right) k_{1, n}+\frac{1}{6 \alpha} k_{2, n}\right), \\
y_{n+1}^{(3)} & =y_{n}^{(3)}+\left(\frac{2 \alpha_{1}-1}{2 \alpha}\right)\left(k_{1, n}-k_{2, n}\right)+k_{3, n} .
\end{aligned}
$$

The information flow is represented in the following diagram:

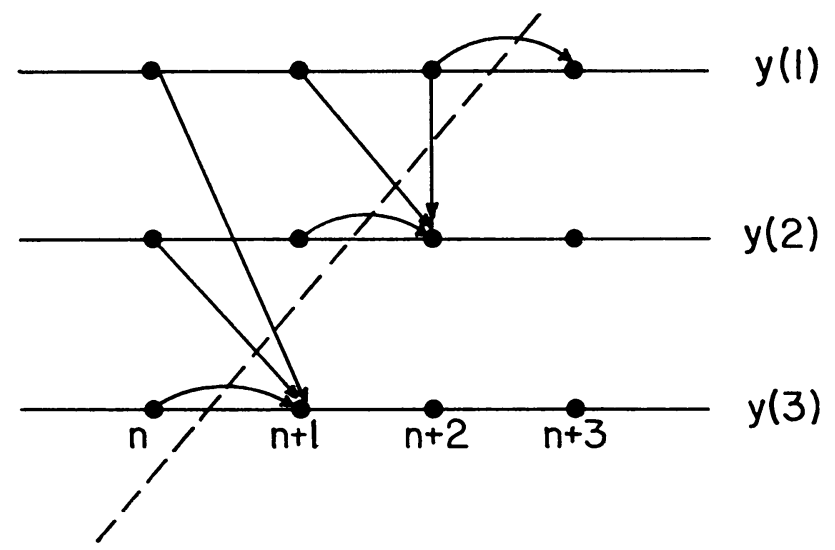

Notice the absence of information flow in an upward direction.

The first four lines of (A.7) represent a parallel second-order Runge-Kutta scheme. (A.7) as a whole, with $\alpha\left(\alpha_{1}\right)$ defined by (A.6), defines a third-order method of the same type. The second-order scheme involves two evaluations of $f$, and some additional arithmetic, which could be carried out simultaneously on two processors. Similarly, the third-order scheme lends itself formally to a three-processor calculation. Unfortunately, the stability analysis for $y^{\prime}=-\lambda y, \lambda=$ const $\geq 0$, shows that both schemes are weakly unstable in the sense that the determinant of the amplification matrix has a double root, respectively a triple root at $z=1$. The schemes thus lead to an error that grows linearly with $n$ as $n \rightarrow \infty$ and $h \rightarrow 0$ for $x_{n}=n h=$ constant. This behavior was confirmed by unfavorable numerical results in a test case.

The difficulty is due to the one-step nature of the formulas with respect to their 
$y$-entries which are the only ones that contribute to the discussion of stability for $h \rightarrow 0$. In the linear multistep methods, this difficulty can be overcome by using formulas whose step number with respect to $y$ is at least two. This makes backcoupling of the predictor to the corrector possible through information lying behind the computation front, and so does not disturb the parallelism. We expect that a similar kind of back-coupling may be devised in the Runge-Kutta case.

IBM Watson Research Center

Yorktown Heights, New York 10598

1. B. Dejon, Numerical Stability of Difference Methods with Matrix Coefficients, RZ 198, Dec. 15,1965

2. C. W. Gear, "Hybrid methods for initial value problems in ordinary differential equations," J. Soc. Indust. Appl. Math. Ser. B. Numer. Anal., v. 2, 1965, pp. 69-86. MR 31 \#3738.

3. Peter Henrici, Error Propagation for Difference Methods, Wiley, New York, 1963. MR 27 \#4365.

4. Peter Henrici, Discrete Variable Methods in Ordinary Differential Equations, Wiley, New York, 1962. MR 24 \#B1772.

5. ZDENĚK Kopal, Numerical Analysis. With Emphasis on the Application of Numerical Techniques to Problems of Infinitesimal Calculus in Single Variable, Wiley, New York; Chapman \& Hall, London, 1955. MR 17, 1007. 